\title{
Static Economic Dispatch for Co-Generation Systems
}

\author{
Frans J. Rooijers, non-member IEEE Robert A.M. van Amerongen, Member IEEE \\ Delft University of Technology, Faculty of Electrical Engineering, Power System Laboratory, \\ Mekelweg 4, 2628 CD DELFT, The Netherlands
}

\begin{abstract}
This paper presents an efficient algorithm for the solution of the economic dispatch of co-generation systems. The key lies in the exploitation of the high degree of separability of the cost function and the constraints. Test results show that for medium size systems (40 units) the method is more than 20 times faster than a conventional procedure based on quadratic programming. Specific implementation details are given in an appendix.
\end{abstract}

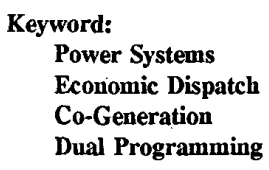

\section{INTRODUCTION}

The economic dispatch problem concerns the question of how to distribute a power load over the units that are in service so that the total fuel cost is at a minimum. In the classic set-up of the problem it is assumed that each unit has a piece-wise linear or quadratic cost function. The optimum distribution is characterized by equal marginal cost for those units that are not fixed at either lower or upper limit. The solution strategy, therefore, is to find that particular value of the marginal cost ("lambda") such that the corresponding electricity production matches the total demand.

A complication arises if one or more units produce both electricity and heat. In this case, not only must the electricity demand be satisfied, but the heat demand as well. In general, with a co-generation system there is one electricity demand and there are a number of distinct heat demands. In figure 1 such a system is schematically depicted. Electricity is produced by both conventional units and co-generation units. In this figure, there are two regions with a heat demand. This

93 SM 468-9 PWRS A paper recommended and approved by the IEEE Power System Engineering Committee of the IEEE Power Engineering Society for presentation at the IEEE/PES 1993 Summer Meeting, Vancouver, B.C., Canada, July 18-22, 1993. Manuscript submitted Dec. 17, 1992; made avallable for printing April 16, 1993.

PRINTED IN USA demand is satisfied by the production of both cogeneration units and additional boiler units. Although in this scheme two heat-regions are shown, the remainder of the paper assumes that there is only one such a region. The method that is presented, however, has no limitation as far as the number of heat demands are concerned.

Basically, the dispatch problem can be formulated as an optimization problem with a quadratic objective function and linear constraints. Such problems can be solved with a general-purpose package that is designed to solve quadratic programming problems, but the computational effort increases at least quadratically with the increasing number of units.

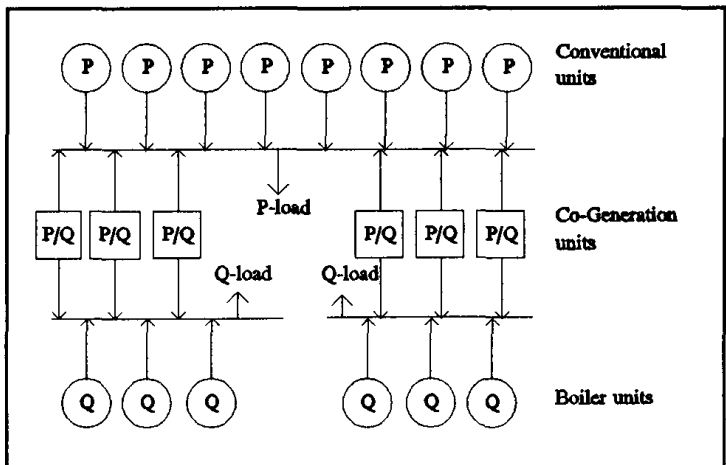

Fig. 1 Schematic picture of a co-generation system. $\mathrm{P}$ and $Q$ are used for electricity and heat respectively.

The problem under consideration, however, has a special feature: separability. The objective function is the sum of cost functions of the separate units. Each constraint, except two, is linked to one specific unit only and thus to one or two variables. There are only two constraints that have system-wide impact: the power balances for both electricity and heat.

Such types of problems are well-suited to be solved with dual, partial-separable, programming (DPS) $)^{1,2}$. In these techniques the main focus is laid on the determination of the multipliers corresponding to the global constraints. The state variables and the multipliers of the local constraints can be obtained very easily once the global multipliers are known.

In our problem, the determination of the state variables is the determination of the production levels of the distinct units. These production levels are found by solving small optimization problems, one problem for 
each unit. The dispatch problem thus reduces to finding that particular value of the multipliers so that the power-balance constraints are satisfied. In this respect, the method is strongly related to the standard economic dispatch problem and to the Lagrange-relaxation based unit commitment procedure ${ }^{3}$.

This paper is organized as follows. In the next section the mathematical problem formulation is given. Section III presents a solution algorithm (with the specific implementation details in an appendix). Numerical results are presented in Section IV.

\section{THE PROBLEM FORMULATION}

The system under consideration has electricity units, co-generation units and heat units. The sets of these units are indicated with $e, c$ and $h$ respectively. A conventional electricity unit $i \in e$ has a convex, quadratic cost function that is given by:

$$
c_{e, i}\left(p_{i}\right)=\alpha_{i}+\beta_{i} * p_{i}+\gamma_{i} * p_{i}^{2}
$$

and the power output $\mathrm{p}_{\mathrm{i}}$ is restricted:

$$
\mathrm{p}_{\mathrm{i}}^{\min } \leq \mathrm{p}_{\mathrm{i}} \leq \mathrm{p}_{\mathrm{i}}^{\max }
$$

A co-generation unit $i \in c$ has a convex cost function in both $p_{i}$ and $q_{i}$. Current practice is that the function is a generalization of a standard quadratic cost function in that terms are added that are linear and quadratic in the heat output. In order to guarantee the dependence between the electricity and the heat productions a socalled coupling-term is added that is linear in both $p_{i}$ and $\mathrm{q}_{\mathrm{i}}$. The form of the cost function thus is:

$$
c_{c, i}\left(p_{i}, q_{i}\right)=\alpha_{i}+\beta_{i} * p_{i}+\gamma_{i} * p_{i}^{2}+\delta_{i} * q_{i}+\epsilon_{i} * q_{i}^{2}+\zeta_{i} * p_{i} * q_{i}
$$

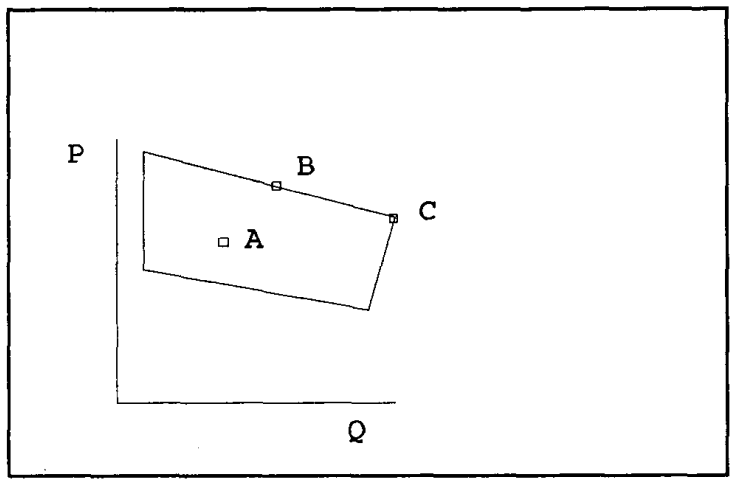

Fig. 2 P-Q diagram of a co-generation unit

The output is assumed to lie in a plane bounded by $n_{i}$ lines. In Figure 2 a typical example of the $P-Q$ diagram is given. The lines are described by:

$$
a_{j i} * p_{i}+b_{j i} * q_{i} \geq c_{j i} \quad j=1, n_{i}
$$

There are three kinds of possible operating points that are characteristic in the solution procedure: in point $A$ the unit is not bounded, in point $B$ the unit is bounded by one constraint and in point $C$ the unit is bounded by two constraints.

A heat production unit $i \in h$ has a convex cost function similar to a conventional unit:

$$
c_{h, i}\left(q_{i}\right)=\alpha_{i}+\delta_{i} * q_{i}+\epsilon_{i} * q_{i}^{2}
$$

and its output $\mathrm{q}_{\mathrm{i}}$ is bounded by:

$$
q_{i}^{\min } \leq q_{i} \leq q_{i}^{\max }
$$

With these data we can formulate the optimization problem. The objective function is the sum of the cost functions of the separate units:

$$
C=\sum_{i \in e} c_{e, i}\left(p_{i}\right)+\sum_{i \in c} c_{c, i}\left(p_{i}, q_{i}\right)+\sum_{i \in h} c_{h, i}\left(q_{i}\right)
$$

The constraints can be divided into two groups: the first group is formed by the global constraints, i.e. the equilibrium constraints of the electricity and the heat production:

$$
\begin{aligned}
& \sum_{i \in e} p_{i}+\sum_{i \in c} p_{i}=p^{\text {demand }} \\
& \sum_{i \in c} q_{i}+\sum_{i \in h} q_{i}=q^{\text {demand }}
\end{aligned}
$$

The second group of constraints is formed by the local constraints: each of them is unique for one unit only. These constraints are given in (2), (4), and (6).

The solution of the optimization problem will satisfy the first-order Kuhn-Tucker conditions. These conditions, which can easily be derived, are given by:

$$
\begin{gathered}
\frac{\partial c_{e, i}}{\partial p_{i}}-\lambda_{p}-\mu_{e, i}=0 \quad \forall i \in e \\
\frac{\partial c_{c, i}}{\partial p_{i}}-\lambda_{p}-\sum_{j} \mu_{c, j i} * a_{j i j}=0 \quad \forall i \in c \\
\frac{\partial c_{c, i}}{\partial q_{i}}-\lambda_{q}-\sum_{j} \mu_{c, j i} * b_{j i}=0 \quad \forall i \in c \\
\frac{\partial c_{h, i}}{\partial q_{i}}-\lambda_{q}-\mu_{h, i}=0 \quad \forall i \in h
\end{gathered}
$$

where:

$\lambda_{\mathrm{p}}$ the multiplier corresponding to the P-load

$\lambda_{\mathrm{q}}$ the multiplier corresponding to the Q-load

$\mu_{e, i}$ the multiplier corresponding to the bound on conventional unit i (2) 
$\mu_{c, i j}$ the multiplier corresponding to the bound $j$ on co-generation unit $i(4)$

$\mu_{h, i}$ the multiplier corresponding to the bound on heat unit $i(6)$

Further, the equality constraints (8) and (9) must be satisfied, and the inequalities (2), (4) and (6) as well. The sign of the multipliers $\mu_{e, i}$ and $\mu_{h, i}$ is an indication of the status of the constraint: if the multiplier is positive, negative or zero then the corresponding constraint is either on its lower bound, or on its upper bound or lies strictly between the bounds. If the multiplier $\mu_{c, j i}$ is positive, then the constraint $j$ of cogeneration unit $i$ is at its bound.

As we assumed convex cost functions, the secondorder optimality condition is always satisfied.

\section{THE SOLUTION SCHEME}

The characteristic point of the problem, and the crucial issue for its solution, is that there are only two system-wide constraints: the two equality constraints (8) and (9) that deal with the power balances. All the other constraints refer to only one unit. This structure naturally leads to a solution procedure where the main focus is laid on the determination of the two multipliers $\lambda_{\mathrm{p}}$ and $\lambda_{\mathrm{q}}$. For every choice of the multipliers, it is very easy to determine the solution that satisfies the optimality conditions (10), (11), (12), (13) and the local constraints (2), (4), (6). This solution is found by solving as many small optimization problems as there are production units. For each type of unit there is a special type of optimization problem.

For the conventional electricity unit ife the objective function to be minimized is:

$$
c_{e, j}\left(p_{i}\right)-\lambda_{p} * p_{i}
$$

where the bound constraint (2) must be taken into account. The solution of this sub-problem satisfies condition (10).

For the co-generation unit $i \in c$ the objective function is:

$$
c_{c, i}\left(p_{i}, q_{i}\right)-\lambda_{p} * p_{i}-\lambda_{q} * q_{i}
$$

During its minimization the constraints (4) must be taken into account. The solution satisfies (11) and (12).

For the heat production unit $i \in h$, finally, the objective function is:

$$
c_{b, i}\left(q_{i}\right)-\lambda_{q} * q_{i}
$$

Constraint (6) must be taken into account. The solution satisfies (13).

Once the two multipliers are known, it is very easy to obtain a solution satisfying all the conditions except the two global constraints (8) and (9). Therefore, the optimization problem can be seen as the problem of how to obtain the multipliers such that the global constraints are satisfied. Because the relation between the multipliers and the production levels and the relation between the change in the multipliers and the change in the production can be obtained very easily, the following procedure can be used:

-0 - set the termination tolerance tol

-1- initialize the multipliers $\lambda_{p}$ and $\lambda_{q}$

-2- determine the electricity and heat production by solving the sub-problem for each unit

-3- determine the production mismatches $\Delta p$ and $\Delta q$

- DO WHILE ( $|\Delta \mathrm{p}|>$ tol or $|\Delta \mathrm{q}|>$ tol $)$

-4- determine (or update) the sensitivity matrix $S$ relating the multiplier changes and the production changes:

$$
\left[S\left[\begin{array}{c}
\Delta \lambda_{\mathrm{p}} \\
\Delta \lambda_{\mathrm{q}}
\end{array}\right]=\left[\begin{array}{c}
\Delta \mathrm{p} \\
\Delta \mathrm{q}
\end{array}\right]\right.
$$

-5 - solve (17) for the change in the multipliers

-6- determine a suitable step length $\alpha$ with $0 \leq \alpha \leq 1$

-7- update the multipliers

$$
\begin{aligned}
& \lambda_{p}:=\lambda_{p}+\alpha * \Delta \lambda_{p} \\
& \lambda_{q}:=\lambda_{q}+\alpha * \Delta \lambda_{q}
\end{aligned}
$$

-8- determine (or update) the production for each unit and the $\mu$-multipliers corresponding to the local constraints

-9- determine the total electricity and heat production and the production mismatches $\Delta p$ and $\Delta \mathrm{q}$

END DO

In this Newton-based iterative process the multipliers are updated such that the final solution is found. A short description of the steps is given here, more detailed information can be found in the appendix.

In the first step the multipliers are initialized. The steps 2 and 3 are trivial. The sensitivity matrix $S$ in step 4 is determined by adding the contribution of each unit. Because during each iteration only one unit undergoes a change in the status of only one of its constraints, the matrix $\mathrm{S}$ must be updated in each iteration instead of re-evaluated. Step 5 is straight- forward. In step 6 a step length is determined. The step size can be restricted by either a move of the multipliers that causes a non-binding constraint to become binding or a move of the multiplier that causes one of the binding constraints to be released from the bound. If the step size is found to be $\alpha=1$ then the problem is solved. In step 7 the multipliers are updated. In step 8 the production and the $\mu$-multipliers are updated. In step 9 the production mismatch is determined. 
The main point of the solution procedure is that there are two levels: at the lower level the production levels are determined when the multipliers are given, at the upper level these multipliers are adapted such that the power production equals the demand.

Seen from an economic point of view ${ }^{4}$, the problem is analogous to that of reaching the optimal overall economy. Theory learns us that this optimum occurs if all the markets are cleared (supply equals demand) while each of the participants is maximizing its own profit. In the problem under consideration there are two markets. Each of the units must be seen as a separate supplier on either the electricity and/or the heat market. Both the multipliers $\lambda_{\mathrm{p}}$ and $\lambda_{\mathrm{q}}$ act as the prices at these markets. At the lower level the separate minimization problems describe the profit-maximizing behavior of the $P$ and/or $Q$ suppliers. The production is determined with given market prices. At the upper level the prices (multipliers) are adapted in the direction of market clearing (mismatch reduction). Once the prices are found such that supply equals the demand, the problem is solved.

Finally, the following remarks are in order:

- in the derivation there is only one heat demand. There is, however, no reason to restrict this number to 1 . If more than 1 demand (or region) is used, then the only complication is that the dimension of (17) increases. The computation time depends, among other things, on the number of units. The number of heat demands is not relevant (except in the solution of (17))

- the procedure basically finds multipliers that correspond to the global constraints. If initial values can be generated (by simple means) that approximate the final values, then the computational effort can be reduced significantly; examples will be given in the next section

- we assumed a single-segmented convex cost function and a convex feasible region for the cogeneration units. It the cost function of either the conventional units or the boiler units is piece-wise quadratic, the method, after a small adaptation, can still be used provided that the marginal cost function is a strictly increasing function of the power output. If segmentation is present in the cost function of cogeneration units, similar requirements must be added to these function in order to guarantee optimality. At present, however, we have no experience with segmented cost function.

If the aggregated cost function is not convex and/or the feasible region is not convex, the procedure outlined in this paper is not guaranteed to yield the optimal solution: a duality gap will occur. This phenomenon is similar to the one that occurs in Lagrange-relaxation based procedures for unit commitment. Our experience, however, is that in practice convexity is used.

- the procedure permits the inclusion of other type of global constraints. Among the possible candidates the inter-area exchange restriction is the most likely one. If such a constraint is added and becomes binding, the set (17) will be expanded to a three-bythree problem . As the number of units for which the constraint holds is always less than the total number of units, regularity of $S$ is guaranteed.

- the procedure in this paper is a static economic dispatch. Applications can be found in standard dispatch problems and as part of a unit commitment procedure. The method can be extended to a dynamic economic dispatch problem where timedependent constraints can be added.

\section{NuMERICAL RESULTS}

In this section we present the results of tests that were carried out to test the performance of the algorithm. As a reference we used a program based on the solution of a quadratic programming problem (Harwell library routine VE02). This reference program was designed to solve dispatch programs with at most 40 units or 60 variables. Both programs were written in Fortran and were executed on an IBM 386-PC.

In the first test we show the computation times of the new algorithm compared with the reference algorithm for several combinations of generation units. The results are given in Table 1 . The number of units is up to 40 , the number of corresponding variables is 56. The ratio between the computation time for the reference algorithm (indicated with QUAD, short for quadratic) and that for the new algorithm (indicated with DPS, short for dual partial separable programming) is given in the column "ratio". It ranges from 2.2 up to 26.9. Not surprisingly, the greater the number of units, the larger this ratio is. This is because the reference program does not exploit the separability and, consequently, uses dense matrices in the objective function and in the constraint specifications.

In the second test we analyze the influence of different combinations of loading levels with a constant set of units. This set comprises 15 conventional power units, 9 boiler and 15 co-generation units. The number of variables is 56. The results are summarized in table 2. From this table it is clear that the advantage of the proposed algorithm is not restricted to a specific loading level. The ratio between computation times is between 17.3 and 72.5 .

Finally, we analyzed the influence of the initial values of the multipliers $\lambda_{p}$ and $\lambda_{q}$ with a constant set of units and different loading levels. The set of units comprises 6 co-generation units, 12 conventional power 
1396

TABLE 1. The performance of the new algorithm in comparison with the reference algorithm for several combination of units

\begin{tabular}{|r|r|r|r|r|r|r|r|r|r||}
\hline Units & Var. & $\begin{array}{r}\text { P-load } \\
(\mathrm{MW})\end{array}$ & $\begin{array}{r}\text { Q-load } \\
(\mathrm{MW})\end{array}$ & $\begin{array}{r}\text { QUAD } \\
(\mathrm{sec})\end{array}$ & ratio & $\begin{array}{r}\text { DPS } \\
(\mathrm{sec})\end{array}$ & iter & final $\lambda_{\mathrm{p}}$ & $\begin{array}{r}\text { final } \\
\lambda_{\mathrm{q}}\end{array}$ \\
\hline 4 & 8 & 300 & 150 & .44 & 2.2 & .21 & 6 & 65.87 & 9.03 \\
6 & 12 & 450 & 300 & 1.24 & 4.9 & .25 & 7 & 66.86 & 11.29 \\
14 & 20 & 1250 & 300 & 3.24 & 10.7 & .30 & 6 & 55.77 & 9.42 \\
25 & 35 & 3500 & 525 & 13.05 & 20.3 & .64 & 8 & 49.58 & 8.44 \\
40 & 56 & 3850 & 700 & 29.23 & 26.9 & 1.09 & 17 & 49.58 & 8.37 \\
\hline
\end{tabular}

TABLE 2. The relative performance of the new algorithm for different load levels. There are 15 conventional, 9 boilers and 15 co-generation units.

\begin{tabular}{|r|r|r|r|r|r|r|r||}
\hline $\begin{array}{r}\text { P-load } \\
(\mathrm{MW})\end{array}$ & $\begin{array}{r}\text { Q-load } \\
(\mathrm{MW})\end{array}$ & $\begin{array}{r}\text { QUAD } \\
(\mathrm{sec})\end{array}$ & ratio & DPS (sec) & iter & final $\lambda_{\mathrm{p}}$ & final $\lambda_{\mathrm{q}}$ \\
\hline 2120 & 300 & 23.35 & 20.2 & 1.16 & 16 & 44.44 & 5.54 \\
2120 & 700 & 26.19 & 30.6 & .85 & 24 & 44.54 & 7.72 \\
2120 & 1050 & 28.30 & 72.5 & .39 & 22 & 44.35 & 17.02 \\
2120 & 1450 & 26.33 & 59.2 & .44 & 14 & 44.31 & 30.53 \\
\hline 3000 & 300 & 23.39 & 24.6 & .95 & 20 & 49.16 & 5.55 \\
3000 & 700 & 28.69 & 30.4 & .94 & 21 & 49.41 & 8.35 \\
3000 & 1050 & 33.47 & 49.3 & .68 & 12 & 49.58 & 17.78 \\
3000 & 1450 & 31.98 & 61.8 & .52 & 14 & 49.58 & 30.53 \\
\hline 3850 & 300 & 21.73 & 17.3 & 1.26 & 9 & 49.58 & 5.60 \\
3850 & 700 & 29.23 & 26.9 & 1.09 & 17 & 49.58 & 8.37 \\
3850 & 1050 & 24.13 & 20.2 & 1.19 & 24 & 68.30 & 18.82 \\
3850 & 1450 & 25.39 & 24.5 & 1.04 & 30 & 68.19 & 30.51 \\
\hline
\end{tabular}

TABLE 3. The performance of the algorithm for several initial values of the multipliers and for several loading levels. There are 12 conventional, 2 boiler and 6 co-generation units

\begin{tabular}{|c|r|r|r|r|r|r|r|r||}
\hline case & initial $\lambda_{\mathrm{p}}$ & initial $\lambda_{\mathrm{q}}$ & $\begin{array}{r}\text { P-load } \\
(\mathrm{MW})\end{array}$ & $\begin{array}{r}\text { Q-load } \\
(\mathrm{MW})\end{array}$ & $\begin{array}{r}\text { DPS } \\
(\mathrm{sec})\end{array}$ & iter & final $\lambda_{\mathrm{p}}$ & final $\lambda_{\mathrm{q}}$ \\
\hline$\Gamma$ & 50.00 & 50.00 & & & 2.14 & 44 & & \\
1 & 50.00 & 0.00 & 1120 & 100 & .85 & 18 & 40.86 & 5.77 \\
$\mathrm{~L}$ & $* 37.53$ & $* 5.01$ & & & .29 & 6 & & \\
$\Gamma$ & 50.00 & 50.00 & & & .76 & 23 & & \\
2 & 50.00 & 0.00 & 1120 & 400 & 2.44 & 43 & 39.63 & 17.98 \\
$\mathrm{~L}$ & $* 37.53$ & $* 20.80$ & & & .23 & 3 & & \\
$\Gamma$ & 50.00 & 50.00 & & & .63 & 19 & & \\
3 & 50.00 & 0.00 & 1120 & 550 & 3.28 & 69 & 39.02 & 33.33 \\
$\mathrm{~L}$ & $* 37.53$ & $* 34.05$ & & & .16 & 3 & & \\
$\Gamma$ & 50.00 & 50.00 & & & 2.03 & 54 & & \\
4 & 50.00 & 0.00 & 2850 & 100 & .71 & 20 & 71.70 & 8.80 \\
$\mathrm{~L}$ & $* 62.26$ & $* 5.01$ & & & .35 & 8 & & \\
$r$ & 50.00 & 50.00 & & & 1.69 & 47 & & \\
5 & 50.00 & 0.00 & 2850 & 400 & 1.37 & 33 & 73.47 & 17.68 \\
$\mathrm{~L}$ & $* 62.26$ & $* 20.80$ & & & .59 & 10 & & \\
$\Gamma$ & 50.00 & 50.00 & & & 1.30 & 36 & & \\
6 & 50.00 & 0.00 & 2850 & 550 & 2.39 & 65 & 73.62 & 33.31 \\
$\mathrm{~L}$ & $* 62.26$ & $* 34.05$ & & & .45 & 11 & & \\
\hline
\end{tabular}


units and 2 boiler units. Table 3 shows the results. For each case we performed tests with 2 manually determined values and with calculated values for the multipliers (indicated with a*). In all cases, the use of calculated values speeded up the solution significantly.

\section{CONCLUSIONS}

In this paper a method is presented to solve the cogeneration static dispatch problem. The proposed methods exploits the special structure of the problem: separability. The solution procedure basically follows a two-level strategy. The method works remarkably fast compared to a conventional procedure. There is neither a limitation of the number of units nor the number of heat demands. Other global constraints can be incorporated easily.

\section{ACKNOWLEDGEMENTS}

The authors wish to express their gratitude to prof. F.A. Lootsma who kindly brought to the authors attention the potential benefits of the dual partialseparable programming technique for the problem under consideration.

\section{REFERENCES}

1 Lootsma, F.A.: "A Comparative Study of Primal and Dual Approaches for Solving Separable and Partially-Separable Non-Linear Optimization Problems', Structural Optimization, vol. 1, pp 7379, 1989

2 Buckers, R.J.L.: "Numerical Experiments with Dual Algorithms for Partially Separable Non-Linear Optimization Problems", in Proceedings of "Parallel Computing 89", North Holland, 1990, pp. 555

3 Merlin, A., P. Sandrin: "A New Method for Unit Commitment at Electricité de France", IEEE Transactions on Power Apparatus and Systems, vol. PAS-102, pp. 1218-1225, 1983

4 Delson, J.K., S.M. Shahidehpour: "Linear Programming Applications to Power System Economics, Planning and Operations", IEEE Transactions on Power Systems, vol. PWRS-7, pp. 1155-1163, 1992

\section{APPENDIX A}

In this appendix we give detailed information on several steps of the algorithm given in Section III. The information refers to the steps 4 (the building of the sensitivity matrix S), 6 (determination of a suitable step length) and 8 (update the production and/or the multipliers corresponding with every unit). It will turn out that each type of unit requires its own special treatment.

The sensitivity matrix $S$ relates changes in multipliers and changes in production for all units together. This matrix is a Jacobian matrix. The general form is:

$$
S=\left[\begin{array}{ll}
\frac{\partial p}{\partial \lambda_{p}} & \frac{\partial p}{\partial \lambda_{q}} \\
\frac{\partial q}{\partial \lambda_{p}} & \frac{\partial q}{\partial \lambda_{q}}
\end{array}\right]
$$

As the production of $p$ and $q$ is the sum of the production of the distinct units, the sensitivity matrix is the sum of the contributions of the separate units. Not every unit will always contribute: an electricity- or heat-production unit does not contribute when its output is at the lower or the upper limit. A co-generation unit does not contribute if two constraints are active (example: point $\mathbf{C}$ in figure 2).

The desired sensitivity relations are obtained by linearizing the relevant Kuhn-Tucker conditions and manipulating them in such a way that changes in production are expressed as a linear function of changes in the $\lambda$-multipliers. The information embedded in these relations can also effectively be used for two other purposes. Firstly, in determining whether the step length must be smaller than 1 , secondly, when updating the solution of the sub-problems, i.e. the production for each unit and the multiplier for any active constraint.

The electricity-production and the heat-production unit are similar with regard to these matters, the cogeneration unit, on the contrary, differs significantly in the way it is handled in the procedure.

For a conventional electricity unit $i \in e$ the linearized (10) yields:

$$
2 \gamma_{i} * \Delta p-\Delta \lambda_{p}-\Delta \mu_{e, i}=0
$$

If the actual power of the unit is not at one of its limits, $\mu_{c, i}$ remains 0 and the contribution to $S$ thus become:

$$
\frac{\Delta \mathrm{p}}{\Delta \lambda_{p}}=\frac{1}{2 \gamma_{1}}
$$

Note that this relation can also be used to determine the step length for a change in the multipliers.

If, on the other hand, the unit is at one of its bounds, a $\lambda_{\mathrm{p}}$ change will not result in a power change and there is no contribution to the sensitivity matrix $S$. The bound-multiplier change is given by:

$$
\Delta \mu_{\mathrm{e}, \mathrm{i}}=-\Delta \lambda_{\mathrm{p}}
$$


Similar formulas can be derived for the heat production units. For a free heat unit, the contribution to $S$ is:

$$
\frac{\Delta q}{\Delta \lambda_{q}}=\frac{1}{2 \varepsilon_{i}}
$$

If the unit is at one of its bounds, the bound-multiplier change is given by:

$$
\Delta \mu_{\mathrm{h}, \mathrm{i}}=-\Delta \lambda_{\mathrm{q}}
$$

For a co-generation unit $i \in c$ things become more complicated. The correct formulas depend on the current state of the unit: the unit has none, one or two active constraints. These situations correspond to the operating points $\mathrm{A}, \mathrm{B}$ and $\mathrm{C}$ of Figure 2 respectively.

If the unit has no active constraints, the linearization of (11) and (12) yields:

$$
\left.\left[\begin{array}{cc}
2 \gamma_{\mathrm{i}} & \zeta_{\mathrm{i}} \\
\zeta_{\mathrm{i}} & 2 \epsilon_{\mathrm{i}}
\end{array}\right] \begin{array}{c}
\Delta \mathrm{p}_{\mathrm{i}} \\
\Delta \mathrm{q}_{\mathrm{i}}
\end{array}\right]=\left[\begin{array}{l}
\Delta \lambda_{\mathrm{p}} \\
\Delta \lambda_{\mathrm{q}}
\end{array}\right]
$$

The inverse of the matrix describes the desired relation between multiplier changes and power changes and this inverse must be added to the sensitivity $\mathrm{S}$. This inverse is also used to determine the direction of the change in the production levels once the multiplier changes are determined. These changes, in turn, are used to check whether one or more of the constraints (4) become violated.

If one constraint of a unit is active, let us say constraint $j$, then this constraint must be considered when linearizing the relevant Kuhn-Tucker conditions:

$$
\left.\left[\begin{array}{ccc}
2 \gamma_{i} & \zeta_{i} & -a_{j i} \\
\zeta_{i} & 2 \epsilon_{i} & -b_{j i} \\
a_{j i} & b_{j i} & 0
\end{array}\right] \begin{array}{c}
\Delta p_{i} \\
\Delta q_{i} \\
\Delta \mu_{c j i j}
\end{array}\right]=\left[\begin{array}{c}
\Delta \lambda_{p} \\
\Delta \lambda_{q} \\
0
\end{array}\right]
$$

The inverse of this matrix gives a relation between the $\lambda$-multipliers on the one hand and the generation output and the $\mu$-multiplier on the other hand. The left-upper $2 \times 2$ part of this inverse must be added to the sensitivity. This inverse matrix is also used for the determination of the step length: once the $\lambda$-multiplier changes are computed, the production changes are used to verify whether one of the constraints, other than $j$, becomes binding. The $\mu$-multiplier change is used to check whether the active constraint $j$ has to be released from its bound.

Finally, if there are two constraints active, for example $\mathrm{j}$ and $\mathrm{k}$, the linearization results in:

$$
\left[\begin{array}{cccc|c}
2 \gamma_{i} & \zeta_{i} & -a_{j i} & -a_{b i} & \Delta p_{i} \\
\zeta_{i} & 2 \varepsilon_{i} & -b_{j i} & -b_{k i} & \Delta q_{i} \\
a_{j i} & b_{j i} & 0 & 0 & \Delta \mu_{c, j i} \\
a_{k j} & b_{k j} & 0 & 0 & \Delta \mu_{c, j i}
\end{array}\right]=\left[\begin{array}{c}
\Delta \lambda_{p} \\
\Delta \lambda_{\mathrm{q}} \\
0 \\
0
\end{array}\right]
$$

From the last two rows, it is seen that a $\lambda$-multiplier change does not result in a production level change. Consequently, there is no contribution to the sensitivity matrix. It is neither reasonable nor necessary to check whether one of the other constraints can become active. It is only required to check whether the step length is restricted because of a sign change in one of the two $\mu$ multipliers. Their change is related to $\lambda$-multiplier changes in the following:

$$
\left[\begin{array}{cc}
-\mathrm{a}_{\mathrm{ji}} & -\mathrm{a}_{\mathrm{ki}} \\
-\mathrm{b}_{\mathrm{ji}} & -\mathrm{b}_{\mathrm{ki}}
\end{array}\left[\begin{array}{l}
\Delta \mu_{\mathrm{c}, \mathrm{ji}} \\
\Delta \mu_{\mathrm{c}, \mathrm{li}}
\end{array}\right]=\left[\begin{array}{c}
\Delta \lambda_{\mathrm{p}} \\
\Delta \lambda_{\mathrm{q}}
\end{array}\right]\right.
$$

These relations are obtained by removing the first two columns and the last two rows of (A.9).

Frans J. Rooijers was born in Haarlem, the Netherlands, on July 22, 1958. He studied electrical engineering at the Delft University of Technology. He received his M.Sc. Electrical Engineering in 1991. From 1983 to 1990 he was coordinator of an energy-saving program for a local government. Since 1990 he works for the Center of Energy Conservation and Environmental Technology (CE) in Delft. $\mathrm{He}$ is manager of the project group Energy. The work of this project group focusses on all aspects of energy resource management and conservation: demand side management and load management, renewable energy systems, efficient energy conversion and (longterm) energy supply.

Robert A.M. van Amerongen (M'88) was born in Heemstede, the Netherlands, on May 3, 1950. He studied electrical engineering at the Delf University of Technology, and economics at the Erasmus University Rotterdam and the University of Amsterdam. He received his M.Sc. Electrical Engineering in 1978 and his Master's in Economics in 1987. In 1978 he entered the Power System Laboratory of the Delft University of Technology as a research assistant. Today he is responsible for education and research. His main areas of interest are electric power-system analysis, including network calculation and its applications, optimization and estimation. 